\title{
LETTER
}

\section{The pooled prevalence of pulmonary embolism in patients with COVID-19}

\author{
Li Shi ${ }^{1}$, Jie Xu ${ }^{1}$, Guangcai Duan ${ }^{1}$, Haiyan Yang ${ }^{1 *}$ (D) and Yadong Wang ${ }^{2^{*}}$
}

๑ 2020 Springer-Verlag GmbH Germany, part of Springer Nature

\section{Dear Editor,}

Shah et al. observed an awfully high prevalence (53.5\%) of pulmonary embolism (PE) among 30 intensive care unit (ICU) patients with coronavirus disease 2019 (COVID-19) in Oxford, UK [1]. Although several studies have focused on this cardiovascular complication of $\mathrm{PE}$ in COVID-19 patients, the prevalence of PE varies from study to study [2-4]. Therefore, we explored the pooled prevalence of PE in COVID-19 patients by a quantitative meta-analysis. Details of our study are shown in Supplementary file 1.

PubMed, EMBASE and Web of Science were reviewed up to August 12th, 2020 to identify relevant studies. Studies reporting the prevalence of confirmed PE in COVID19 patients and with the sample size $\geq 30$ were included. The pooled prevalence and corresponding $95 \%$ confidence interval $(\mathrm{CI})$ were used to assess the combined effects. An additional analysis comparing the prevalence of PE in COVID-19 patients admitted to ICU and nonICU was conducted. Heterogeneity between studies was estimated with $I^{2}$ statistic and Cochran's Q (reported as $\chi^{2}$ and $P$ values) [5]. Subgroup analysis and meta-regression analysis were conducted by country, study design, sample size, quality score, PE diagnosis and prevalence of prophylactic anticoagulation to explore possible sources of heterogeneity.

Among 1981 potentially related studies, 49 articles (52 studies) comprising 20,523 COVID-19 patients were enrolled in this meta-analysis after rigorous screening

\footnotetext{
*Correspondence: yhy@zzu.edu.cn; wangyd76@163.com ${ }^{1}$ Department of Epidemiology, College of Public Health, Zhengzhou University, No. 100 of Science Avenue, Zhengzhou 450001, China 2 Department of Toxicology, Henan Center for Disease Control and Prevention, No. 105 of South Nongye Road, Zhengzhou 450016, China

Full author information is available at the end of the article
}

(Suppl. File 2, Fig. S1). The principal characteristics and details about the PE diagnosis of the included studies were shown in Supplementary file 2, Table S4 and Table S5. The pooled prevalence of PE in COVID-19 patients was $8 \%\left(95 \%\right.$ CI $6-11 \% ; X^{2}=1259.68, P<0.01$; $I^{2}=95.95 \%$; random-effects model) (Fig. 1a). Due to the obvious heterogeneity, we performed subgroup analysis and meta-regression. None of these factors we explored further was significantly correlated with the interstudy heterogeneity on subgroup analysis (Suppl. File 2, Table S6). However, the results of meta-regression indicated that sample size $(P=0.019)$ and the proportion of patients undergoing PE diagnosis $(P<0.001)$ might be potential sources of heterogeneity (Suppl. File 2, Table S6). The pooled prevalence of PE in patients undergoing PE diagnosis was $28 \%$ (95\% CI $22-34 \% ; \chi^{2}=429.11$, $P<0.01 ; I^{2}=93.71 \%$; random-effects model) on the basis of 28 studies consisting of 4387 patients undergoing PE diagnosis (Fig. 1b). The significantly higher pooled prevalence of PE was observed in COVID-19 patients admitted to ICU $\left(19 \%, 95 \%\right.$ CI $14-25 \%$; $\chi^{2}=346.07, P<0.01$; $\left.I^{2}=92.49 \%\right)$ compared with those admitted to non-ICU (9\%, 95\% CI 6-13\%; $\left.X^{2}=379.37, P<0.01 ; I^{2}=94.99 \%\right)$ (Fig. 1c, d). The Begg's test $(P=0.002)$ and Egger's test $(P<0.001)$ suggested that potential publication bias existed within our analysis.

In summary, it is needed to pay more attention to the relatively high prevalence of PE in COVID-19 patients, especially in ICU wards. Future studies that will explore the detection method considering high infectivity of COVID-19 and antithrombotic treatment balancing the risk of thrombosis and the risk of bleeding are needed.

\section{Electronic supplementary material}

The online version of this article (https://doi.org/10.1007/s00134-020-06235-8) contains supplementary material, which is available to authorized users.

\section{Springer}




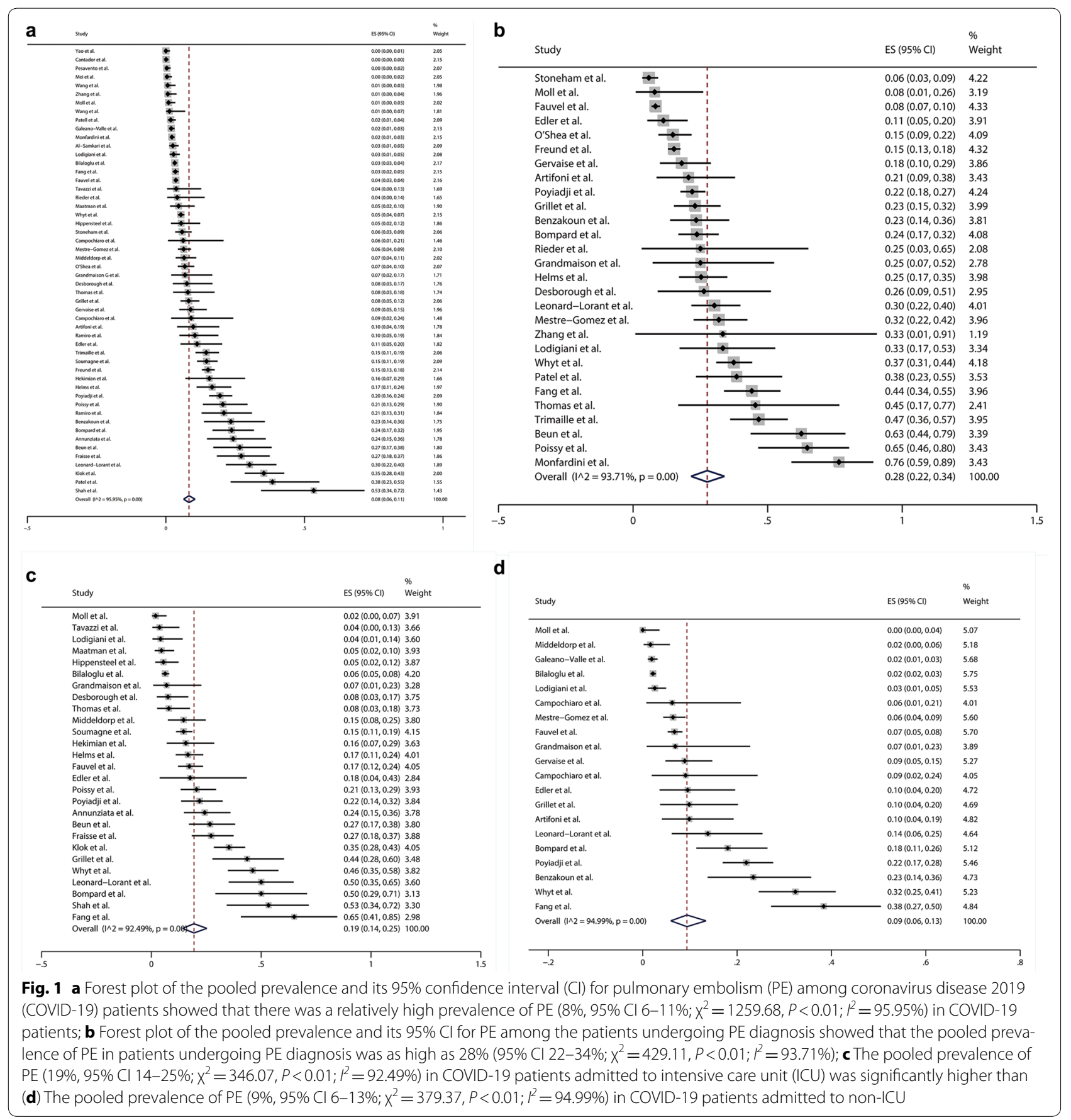

\section{Author details}

${ }_{1}^{1}$ Department of Epidemiology, College of Public Health, Zhengzhou University, No. 100 of Science Avenue, Zhengzhou 450001, China. ${ }^{2}$ Department of Toxicology, Henan Center for Disease Control and Prevention, No. 105 of South Nongye Road, Zhengzhou 450016, China.

\section{Acknowledgements}

We would like to thank Ying Wang, Xuan Liang, Wenwei Xiao, Peihua Zhang and Jian Wu (All are from Department of Epidemiology, College of Public Health, Zhengzhou University) for their kind help in searching articles and collecting data, and valuable suggestions for data analysis.

\section{Author contributions}

$L S, H Y$, and $Y W$ conceptualized the study. LS and JX extracted the data. LS, JX, and $Y W$ analyzed the data. $L S, G D, H Y$, and $Y W$ contributed to the methodology. LS, HY, and YW wrote and reviewed the manuscript. All the authors approved the final manuscript.

\section{Funding}

This work was supported by grants from the National Natural Science Foundation of China (Grant Number 81973105), the National Science and Technology Major Projects of China (Grant Number 2018ZX10301407) and Joint Construction Project of Henan Medical Science and Technology Research Plan (Grant 
Number LHGJ20190679). The funders have no role in the data collection, data analysis, preparation of manuscript and decision to submission.

\section{Data availability}

All data relevant to the study are included in the article or uploaded as supplementary information.

\section{Compliance with ethical standards}

\section{Conflicts of interest}

The authors declare that they have no any potential conflict of interest regarding this submitted manuscript.

\section{Publisher's Note}

Springer Nature remains neutral with regard to jurisdictional claims in published maps and institutional affiliations.

Accepted: 29 August 2020

Published online: 14 September 2020

\section{References}

1. Shah A, Frost JN, Aaron L, Donovan K, Drakesmith H, Collaborators (2020) Systemic hypoferremia and severity of hypoxemic respiratory failure in COVID-19. Crit Care 24:320. https://doi.org/10.1186/s13054-020-03051-w

2. Pesavento R, Ceccato D, Pasquetto G et al (2020) The hazard of (sub) therapeutic doses of anticoagulants in non-critically ill patients with Covid-19: the Padua province experience. J Thromb Haemost. https://doi. org/10.1111/jth.15022

3. Hekimian G, Lebreton G, Brechot N, Luyt CE, Schmidt M, Combes A (2020) Severe pulmonary embolism in COVID-19 patients: a call for increased awareness. Crit Care 24:274. https://doi.org/10.1186/s13054-020-02931-5

4. Fang C, Garzillo G, Batohi B et al (2020) Extent of pulmonary thromboembolic disease in patients with COVID-19 on CT: relationship with pulmonary parenchymal disease. Clin Radiol. https://doi.org/10.1016/j. crad.2020.07.002

5. Higgins JP, Thompson SG, Deeks JJ, Altman DG (2003) Measuring inconsistency in meta-analyses. BMJ 327:557-560. https://doi.org/10.1136/ bmj.327.7414.557 